\title{
Orbital Stability of Peakons for a Generalized Camassa-Holm Equation
}

\author{
Xinhui Lu', Aiyong Chen',2, Tongjie Deng1 \\ ${ }^{1}$ Department of Mathematics and Computing Science, Guilin University of Electronic Technology, Guilin, China \\ ${ }^{2}$ Department of Mathematics, Hunan First Normal University, Changsha, China \\ Email: xiaoxiaolui@163.com, aiyongchen@163.com,2722699406@qq.com
}

How to cite this paper: Lu, X.H., Chen, A.Y. and Deng, T.J. (2019) Orbital Stability of Peakons for a Generalized Camassa-Holm Equation. Journal of Applied Mathematics and Physics, 7, 2200-2211.

https://doi.org/10.4236/jamp.2019.710151

Received: September 1, 2019

Accepted: October 5, 2019

Published: October 8, 2019

Copyright $\odot 2019$ by author(s) and Scientific Research Publishing Inc. This work is licensed under the Creative Commons Attribution International License (CC BY 4.0).

http://creativecommons.org/licenses/by/4.0/

\begin{abstract}
We investigate the orbital stability of the peakons for a generalized Camassa-Holm equation ( $\mathrm{gCH}$ ). Using variable transformation, a planar dynamical system is obtained from the $\mathrm{gCH}$ equation. It is shown that the planar system has two heteroclinic cycles which correspond two peakon solutions. We then prove that the peakons for the $\mathrm{gCH}$ equation are orbitally stable by using the method of Constantin and Strauss.
\end{abstract}

\section{Keywords}

Camassa-Holm Equation, Peakon, Heteroclinic Orbit, Orbital Stability

\section{Introduction}

In recent years, there has been great interest in the nonlinearly dispersive equations for model breaking waves. The first widely studied equation of this kind is Camassa-Holm $(\mathrm{CH})$ equation [1].

$$
u_{t}-u_{t x x}-3 u u_{x}+2 u_{x} u_{x x}+u u_{x x x}=0
$$

for $u(x, t)$. This equation arises from shallow water waves theory [1] [2] and provides a wave breaking model for a large class of solutions in which the wave slope blows up in a finite time while the wave amplitude remains bounded [3] [4] [5] [6]. A special class of weak solutions of the equation describes the solitary waves at the peaked, called peakons [1] [7] [8], whose wave slope is discontinuous at the wave peak. More noteworthy, the $\mathrm{CH}$ equation is an integrable system [1] [9] [10], possessing a Lax pair, a bi-Hamiltonian structure, and an infinite hierarchy of symmetries and conservation laws.

An interesting nonlinear generalization of the $\mathrm{CH}$ equation $(\mathrm{gCH})$

$$
u_{t}-u_{x x t}=\frac{1}{2}(p+1)(p+2) u^{p} u_{x}-\frac{1}{2} p(p-1) u^{p-2} u_{x}^{3}-2 p u^{p-1} u_{x} u_{x x}-u^{p} u_{x x x} \text {, }
$$


was discussed in [11]. When $p=1$, then Equation (2) is the classical CH Equation (1).

Similar to the $\mathrm{CH}$ equation, the gCH Equation (2) is also the form of conservation law

$$
m_{t}-\left(\frac{1}{2} p u^{p-1}\left(u^{2}-u_{x}^{2}\right)+u^{p} m\right)_{x}=0, m=u-u_{x x} .
$$

Therefore, integral

$$
P=\int_{-\infty}^{+\infty} m \mathrm{~d} x
$$

is conserved (under the appropriately asymptotic decay condition on $u$ ). The other conservation integral is given by the Hamiltonian

$$
E_{(p)}=\int_{-\infty}^{+\infty} \frac{1}{2} u^{p}\left(u^{2}+u_{x}^{2}\right) \mathrm{d} x, \quad p \neq 0 .
$$

This integral leads to the conservation law

$$
D_{t} T+D_{x} X=0
$$

with

$$
\begin{gathered}
T=\frac{1}{2} u^{p}\left(u^{2}+u_{x}^{2}\right), \\
X=-u^{p} u_{t} u_{x}+\frac{1}{2}(\delta E / \delta m)^{2}-\frac{1}{2}\left(D_{x}(\delta E / \delta m)\right)^{2},
\end{gathered}
$$

where

$$
\delta E / \delta m=\Delta^{-1}(\delta E / \delta u)=\Delta^{-1}\left(\frac{1}{2} p u^{p-1}\left(u^{2}-u_{x}^{2}\right)+u^{p}\left(u-u_{x x}\right)\right)
$$

and $\Delta=1-D_{x}^{2}$. The complete classification of gCH Equation (2) conservation laws has been given [11].

Without loss of generality, let $p=2$, then Equation (2) becomes

$$
u_{t}-u_{t x x}-\left(u\left(u^{2}-u_{x}^{2}\right)+u^{2}\left(u-u_{x x}\right)\right)_{x}=0
$$

Equation (9) has the peakon solution

$$
u(x, t)=c \varphi(x+c t)=c \mathrm{e}^{-|x+c t|}, \quad c \in \mathbb{R} .
$$

As Constantin and Strauss said in [12], the peakons are solitons and therefore their sizes and velocities do not change as a result of collision, so that it is reasonable to expect that they are stable. Because a small perturbation of a solitary wave can yield another one with a different speed and phase shift, the appropriate notion of stability is orbital stability. That is, a wave starting close to a solitary wave remains close to some translate of it at all later times. Thus the shape of the wave remains approximately the same for all times. The peakons were proved to be orbitally stable by Constantin and Strauss in [12]. The approach in [13] was extended to prove the orbital stability of the peakons for the other nonlinear wave equations [14]-[25]. The method of proved orbital stable of peakons was also extended to periodic peakons [26] [27] [28] [29] [30]. In [11], Anco and 
Recio obtained an interesting generalization of the Camassa-Holm and FORQ/modified Camassa-Holm equations by deriving the most general subfamily of peakon equations that possess the Hamiltonian structure shared by the Camassa-Holm and FORQ/modified Camassa-Holm equations. They showed that Equation (2) admits peakons. As a special case of Equation (2), Equation (9) is a generalized $\mathrm{CH}$ equation with cubic nonlinearity. To the best of our knowledge, the orbital stability of peakons of Equation (9) is not considered. In this paper, we will prove the orbital stability of peakons of the gCH Equation (9).

Theorem 1 For every $\epsilon>0$, there is a $\delta>0$ such that if $u \in C\left([0, T) ; H^{1}(\mathbb{R})\right)$ is a solution to $(9)$ with

$$
\|u(\cdot, 0)-\varphi\|_{H^{1}(\mathbb{R})}<\delta,
$$

then

$$
\|u(\cdot, t)-\varphi(\cdot-\xi(t))\|_{H^{1}(\mathbb{R})}^{2}<\epsilon .
$$

for $t \in(0, T)$, where $\xi(t) \in \mathbb{R}$ is any point where the function $u(\cdot, t)$ attains its maximum.

The remaining part is organized as follows. In Section 2, we analyze the peakon solutions of the gCH equation by using dynamical system method. In Section 3, the orbital stability of peakon is proved. A short conclusion is given in Section 4

\section{Peakon Solutions of the gCH Equation}

In this section, we convert Equation (9) into a planar dynamical system. By substituting $u(x, t)=\varphi(\tau)$ with $\tau=x+c t$ into Equation (9), then it follows that

$$
c \varphi^{\prime}-c \varphi^{\prime \prime \prime}-\left(2 \varphi^{3}-\varphi\left(\varphi^{\prime}\right)^{2}-\varphi^{2} \varphi^{\prime \prime}\right)=0
$$

where $\varphi^{\prime}$ is the derivative with respect to $\tau$. Integrating Equation (13) once we obtain

$$
c \varphi-c \varphi^{\prime \prime}-\left(2 \varphi^{3}-\varphi\left(\varphi^{\prime}\right)^{2}-\varphi^{2} \varphi^{\prime \prime}\right)=g
$$

where $g$ is the integral constant. Letting $y=\frac{\mathrm{d} \varphi}{\mathrm{d} \tau}$, then we obtain the following planar dynamical system

$$
\left\{\begin{array}{l}
\frac{\mathrm{d} \varphi}{\mathrm{d} \tau}=y, \\
\frac{\mathrm{d} y}{\mathrm{~d} \tau}=\frac{-\varphi y^{2}+2 \varphi^{3}-c \varphi+g}{\varphi^{2}-c},
\end{array}\right.
$$

with first integral

$$
H(\varphi, y)=\left(\varphi^{2}-c\right)\left[y^{2}-\varphi^{2}-\frac{2 g \varphi}{\varphi^{2}-c}\right]=h .
$$


As $g=0$, we have the following planar system

$$
\left\{\begin{array}{l}
\frac{\mathrm{d} \varphi}{\mathrm{d} \tau}=y, \\
\frac{\mathrm{d} y}{\mathrm{~d} \tau}=\frac{-\varphi y^{2}+2 \varphi^{3}-c \varphi}{\varphi^{2}-c},
\end{array}\right.
$$

with first integral

$$
H(\varphi, y)=\left(\varphi^{2}-c\right)\left(y^{2}-\varphi^{2}\right)
$$

On the singular line $\varphi^{2}=c$, system (17) is discontinuous. To avoid the line temporarily we make transformation $\mathrm{d} \tau=\left(\varphi^{2}-c\right) \mathrm{d} \zeta$. Under this transformation, system (17) becomes

$$
\left\{\begin{array}{l}
\frac{\mathrm{d} \varphi}{\mathrm{d} \zeta}=\left(\varphi^{2}-c\right) y, \\
\frac{\mathrm{d} y}{\mathrm{~d} \zeta}=-\varphi y^{2}+2 \varphi^{3}-c \varphi .
\end{array}\right.
$$

System (17) and (19) have the same first integral as (18). Consequently, system (19) has the same topological phase portraits as system (17) except for the singular line $\varphi^{2}=c$. Obviously, $\varphi^{2}=c$ is an invariant straight-line solution for system (19). When $c>0$, there are three equilibrium points $O(0,0)$ and $C_{1,2}\left( \pm \frac{\sqrt{2 c}}{2}, 0\right)$ of system (19). In addition, there are four equilibrium points $S_{1,2}(\sqrt{c}, \pm \sqrt{c})$ and $S_{3,4}(-\sqrt{c}, \pm \sqrt{c})$ on the straight line $\varphi^{2}=c$.

Let $M\left(\varphi_{e}, y_{e}\right)$ be the coefficient matrix of the linearized system (19) at equilibrium point $\left(\varphi_{e}, y_{e}\right)$ and define $J=\operatorname{det} M\left(\varphi_{e}, y_{e}\right)$. Then $J_{O}=-c^{2}<0$, $J_{C_{1,2}}=c^{2}>0$ and $J_{S_{1,2,3,4}}=-4 c^{2}<0$. By the theory of planar dynamical systems, we know that for an equilibrium point of a planar integrable system, if $J<0$ then the equilibrium point is a saddle point; if $J>0$ then it is a center point; if $J=0$ and the Poincaré index of the equilibrium point is 0 then is a cusp, otherwise, it is a high order equilibrium point. Therefore, $O$ and $S_{1,2,3,4}$ are five saddle points and $C_{1,2}$ are two center points. Phase portrait of the system (17) is shown in Figure 1 (a). Note that the algebraic curves defined by $H(\varphi, y)=0$ consists of two heteroclinic cycles (see Figure 1(b)), the two heteroclinic cycles correspond to two peakons.

The algebraic curve defined by $H(\varphi, y)=0$ gives

$$
y^{2}=\varphi^{2}
$$

By using the first equation of system (17) to do the integration, we obtain the peakon solutions with exponential function form

$$
\varphi(x)= \pm \sqrt{c} \mathrm{e}^{-|x|}
$$

The profiles of peakons are shown in Figure 2.

Definition 1 Given initial data $u_{0} \in W^{1,3}(\mathbb{R})$, the function $u \in L^{\infty}\left([0, T], W^{1,3}(\mathbb{R})\right)$ is said to be a weak solution to (9) if it satisfies the following identity: 


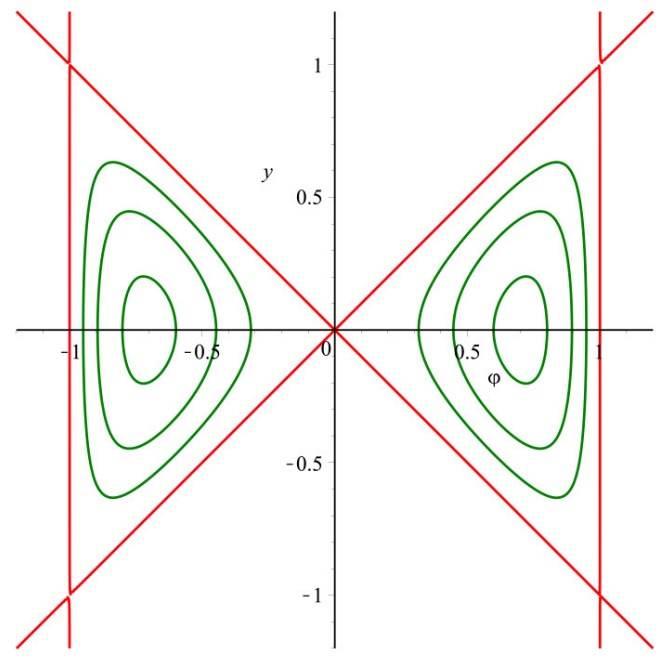

(a)

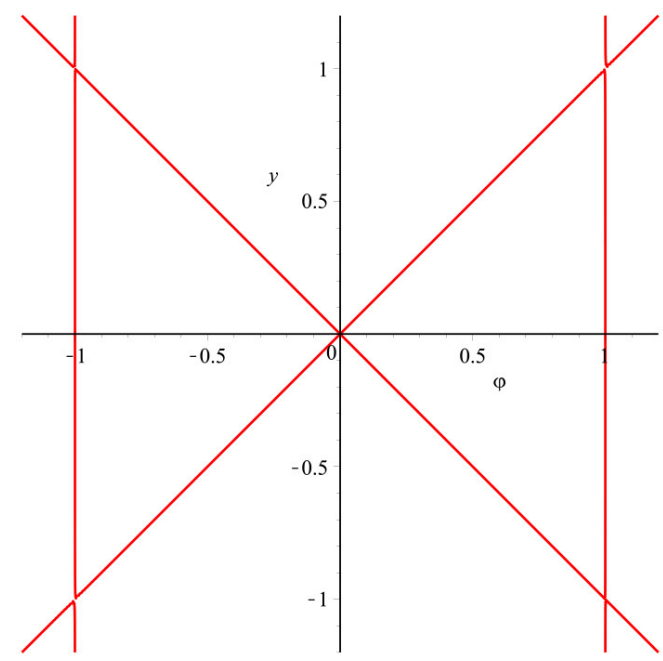

(b)

Figure 1. (Color online.) Phase portrait and algebraic curve. (a) Phase portrait of the system (17); (b) The algebraic curve defined by $H(\varphi, y)=0$.

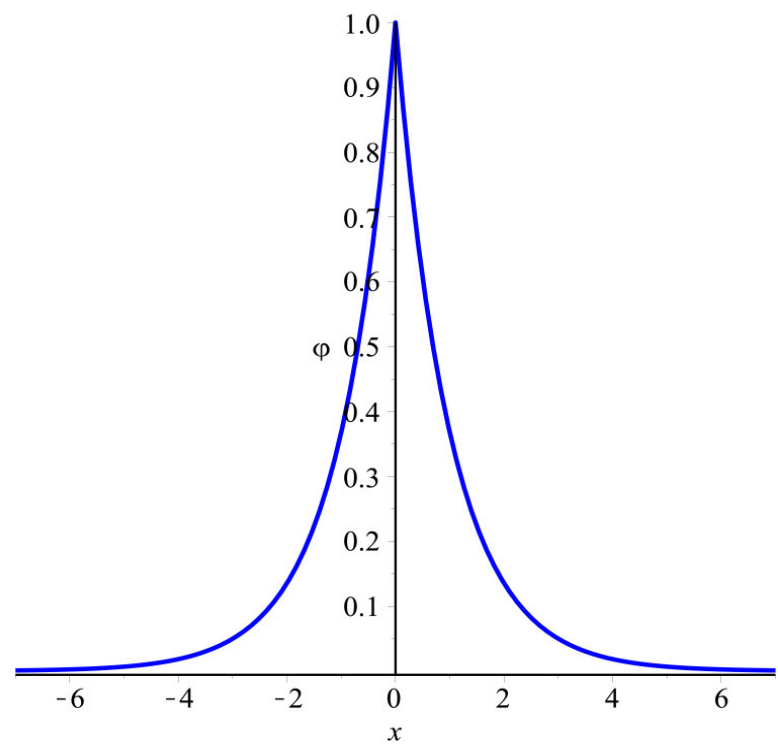

(a)

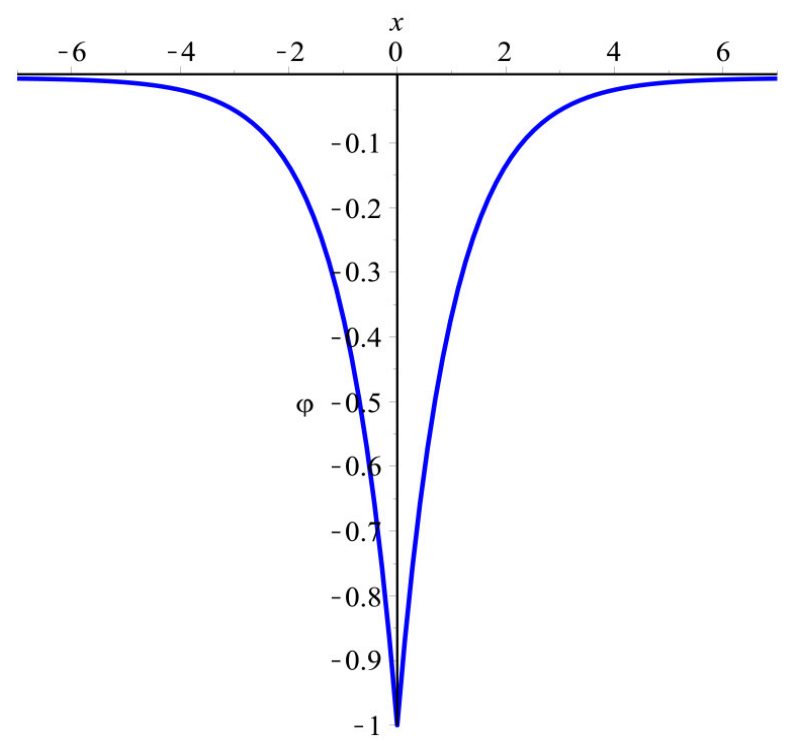

(b)

Figure 2. (Color online.) The profile of peakon for $c=1$.

$$
\int_{0}^{T} \int_{\mathbb{R}}\left[u \psi_{t}-\frac{1}{3} u^{3} \psi_{x}-p *\left(\frac{5}{3} u^{3}+u u_{x}^{2}\right) \psi\right] \mathrm{d} x \mathrm{~d} t+\int_{\mathbb{R}} u(x, 0) \psi(x, 0) \mathrm{d} x=0 .
$$

for any smooth test function $\varphi(t, x) \in C_{c}^{\infty}([0, T] \times \mathbb{R})$. If $u$ is a weak solution on $[0, T)$ for every $T>0$, then it is called a global weak solution.

Theorem 2 [11] The peaked functions of the form

$$
u(t, x)= \pm \sqrt{c} \mathrm{e}^{-|x+c t|},
$$


is a global weak solution to (9), in the sense of Definition 1.

\section{Proof of Stability}

Note that a small change in the shape of a peakon can yield another one with a different speed. The appropriate notion of stability is, therefore, that of orbital stability: a wave with an initial profile close to a peakon remains close to some translate of it for all later times. That is, the shape of the wave remains approximately the same for all times.

Equation (9) has the conservation laws

$$
H_{1}[u]=\int_{\mathbb{R}}\left(u^{2}+u_{x}^{2}\right) \mathrm{d} x, \quad H_{2}[u]=\int_{\mathbb{R}}\left(u^{4}+u^{2} u_{x}^{2}\right) \mathrm{d} x .
$$

For the convenience of calculation, we take $c=1$, then $\varphi(x)=\mathrm{e}^{-|x|}$. Using (24), we obtain

$$
H_{1}[\varphi]=\int_{\mathbb{R}}\left(\varphi^{2}+\varphi_{x}^{2}\right) \mathrm{d} x=2, \quad H_{2}[\varphi]=\int_{\mathbb{R}}\left(\varphi^{4}+\varphi^{2} \varphi_{x}^{2}\right) \mathrm{d} x=1 .
$$

We next consider the expansion of the conservation law $H_{1}$ around the peakon $\varphi$ in the $H^{1}(\mathbb{R})$-norm.

Lemma 1 For every $u \in H^{1}(\mathbb{R})$ and $\xi \in \mathbb{R}$,

$$
H_{1}[u]-H_{1}[\varphi]=\|u-\varphi(\cdot-\xi)\|_{H^{1}(\mathbb{R})}^{2}+4(u(\xi)-1) .
$$

Proof. we calculate

$$
\begin{aligned}
& \|u-\varphi(\cdot-\xi)\|_{H^{1}(\mathbb{R})}^{2} \\
& =H_{1}[u]+H_{1}[\varphi]-2 \int_{\mathbb{R}} u_{x}(x) \varphi_{x}(x-\xi) \mathrm{d} x-2 \int_{\mathbb{R}} u(x) \varphi(x-\xi) \mathrm{d} x \\
& =H_{1}[u]+H_{1}[\varphi]-2 \int_{-\infty}^{\xi} u_{x}(x) \varphi_{x}(x-\xi) \mathrm{d} x-2 \int_{\xi}^{+\infty} u_{x}(x) \varphi_{x}(x-\xi) \mathrm{d} x \\
& \quad-2 \int_{\mathbb{R}} u(x) \varphi(x-\xi) \mathrm{d} x .
\end{aligned}
$$

Since

$$
\int_{-\infty}^{\xi} u_{x}(x) \varphi_{x}(x-\xi) \mathrm{d} x=u(\xi)-\int_{-\infty}^{\xi} u(x) \varphi(x-\xi) \mathrm{d} x,
$$

and

$$
\int_{\xi}^{+\infty} u_{x}(x) \varphi_{x}(x-\xi) \mathrm{d} x=u(\xi)-\int_{\xi}^{+\infty} u(x) \varphi(x-\xi) \mathrm{d} x .
$$

Therefore, we have

$$
\|u-\varphi(\cdot-\xi)\|_{H^{1}(\mathbb{R})}^{2}=H_{1}[u]-H_{1}[\varphi]+4(1-u(\xi)) .
$$

This proves the lemma.

Lemma 2 For every $u \in H^{1}(\mathbb{R})$, let $M=\max _{x \in \mathbb{R}}\{u(x)\}$. Then

$$
H_{2}[u] \leq M^{2} H_{1}[u]-M^{4} .
$$

Proof. Let $\xi \in \mathbb{R}$ be such that $M=u(\xi)$ and define

$$
g(x)= \begin{cases}u(x)-u_{x}(x), & x<\xi, \\ u(x)+u_{x}(x), & x>\xi .\end{cases}
$$


We calculate

$$
\begin{aligned}
\int_{\mathbb{R}} g^{2}(x) \mathrm{d} x & =\int_{-\infty}^{\xi}\left[u(x)-u_{x}(x)\right]^{2} \mathrm{~d} x+\int_{\xi}^{+\infty}\left[u(x)+u_{x}(x)\right]^{2} \mathrm{~d} x \\
& =\int_{\mathbb{R}}\left[u^{2}(x)+u_{x}^{2}(x)\right] \mathrm{d} x-\left.u^{2}(x)\right|_{-\infty} ^{\xi}+\left.u^{2}(x)\right|_{\xi} ^{+\infty} \\
& =H_{1}[u]-2 M^{2} .
\end{aligned}
$$

Similarly,

$$
\begin{aligned}
& \int_{\mathbb{R}} u^{2} g^{2}(x) \mathrm{d} x \\
& =\int_{-\infty}^{\xi} u^{2}\left[u(x)-u_{x}(x)\right]^{2} \mathrm{~d} x+\int_{\xi}^{+\infty} u^{2}\left[u(x)+u_{x}(x)\right]^{2} \mathrm{~d} x \\
& =\int_{\mathbb{R}}\left[u^{4}+u^{2} u_{x}^{2}\right] \mathrm{d} x-\left.\frac{1}{2} u^{4}(x)\right|_{-\infty} ^{\xi}+\left.\frac{1}{2} u^{4}(x)\right|_{\xi} ^{+\infty} \\
& =H_{2}[u]-M^{4} .
\end{aligned}
$$

Since

$$
\int_{\mathbb{R}} u^{2} g^{2}(x) \mathrm{d} x \leq M^{2} \int_{\mathbb{R}} g^{2}(x) \mathrm{d} x,
$$

we get

$$
H_{2}[u] \leq M^{2} H_{1}[u]-M^{4} .
$$

This thus completes the proof of Lemma 2.

Lemma 3 For every $u \in H^{1}(\mathbb{R})$, if $\|u-\varphi\|_{H^{1}}<\delta$, then

$$
\left|H_{1}[u]-H_{1}[\varphi]\right| \leq \delta(\delta+2 \sqrt{2})
$$

and

$$
\left|H_{2}[u]-H_{2}[\varphi]\right| \leq \delta\left(\frac{1}{2} \delta^{3}+2 \sqrt{2} \delta^{2}+6 \delta+4 \sqrt{2}\right) .
$$

Proof. Identity (33) shows that for all $v \in H^{1}(\mathbb{R})$,

$$
\sup _{x \in \mathbb{R}}|v(x)| \leq \sqrt{\frac{1}{2} H_{1}[v]}=\frac{\sqrt{2}}{2}\|v\|_{H^{1}} .
$$

Equality holds if and only if $v$ is proportional to a translate of $\varphi$. Note that

$$
\begin{aligned}
\left|H_{1}[u]-H_{1}[\varphi]\right| & =\left|\left(\|u\|_{H^{1}}+\|\varphi\|_{H^{1}}\right)\left(\|u\|_{H^{1}}-\|\varphi\|_{H^{1}}\right)\right| \\
& \leq\left(\|u-\varphi\|_{H^{1}}+2\|\varphi\|_{H^{1}}\right)\|u-\varphi\|_{H^{1}} \\
& \leq \delta(\delta+2 \sqrt{2}) .
\end{aligned}
$$

Similarly,

$$
\begin{aligned}
& \left|H_{2}[u]-H_{2}[\varphi]\right| \\
& =\left|\int_{\mathbb{R}}\left(u^{2}-\varphi^{2}\right)\left(u^{2}+u_{x}^{2}\right) \mathrm{d} x+\int_{\mathbb{R}} \varphi^{2}\left(u^{2}+u_{x}^{2}-\varphi^{2}-\varphi_{x}^{2}\right) \mathrm{d} x\right| \\
& =\mid \int_{\mathbb{R}}\left(u^{2}-\varphi^{2}\right)\left(u^{2}+u_{x}^{2}\right) \mathrm{d} x+\int_{\mathbb{R}} \varphi^{2}\left[(u-\varphi)^{2}+\left(u_{x}-\varphi_{x}\right)^{2}\right] \mathrm{d} x \\
& \quad+\int_{\mathbb{R}} \varphi^{2}\left[2(u-\varphi) \varphi+2\left(u_{x}-\varphi_{x}\right) \varphi_{x}\right] \mathrm{d} x \mid .
\end{aligned}
$$


Since

$$
\begin{aligned}
& \left|\int_{\mathbb{R}}\left(u^{2}-\varphi^{2}\right)\left(u^{2}+u_{x}^{2}\right) \mathrm{d} x\right| \\
& \leq\left(\|u\|_{L^{\infty}}+\|\varphi\|_{L^{\infty}}\right)\|u-\varphi\|_{L^{\infty}} H_{1}[u] \\
& \leq \frac{1}{2}\left(\|u\|_{H^{1}}-\|\varphi\|_{H^{1}}+2 \sqrt{2}\right)\|u-\varphi\|_{H^{1}} H_{1}[u] \\
& \leq \frac{1}{2} \delta(\delta+2 \sqrt{2})\left(2+2 \sqrt{2} \delta+\delta^{2}\right)
\end{aligned}
$$

and

$$
\begin{aligned}
& \left|\int_{\mathbb{R}} \varphi^{2}\left[(u-\varphi)^{2}+\left(u_{x}-\varphi_{x}\right)^{2}\right] \mathrm{d} x+\int_{\mathbb{R}} \varphi^{2}\left[2(u-\varphi) \varphi+2\left(u_{x}-\varphi_{x}\right) \varphi_{x}\right] \mathrm{d} x\right| \\
& \leq\left\|\varphi^{2}\right\|_{L^{\infty}}\|u-\varphi\|_{H^{1}}^{2}+2\left\|\varphi^{2}\right\|_{L^{\infty}}\|u-\varphi\|_{H^{1}}\|\varphi\|_{H^{1}} \\
& \leq \delta^{2}+2 \sqrt{2} \delta,
\end{aligned}
$$

then

$$
\begin{aligned}
\left|H_{2}[u]-H_{2}[\varphi]\right| & \leq \frac{1}{2} \delta(\delta+2 \sqrt{2})\left(2+2 \sqrt{2} \delta+\delta^{2}\right)+\delta^{2}+2 \sqrt{2} \delta \\
& =\delta\left(\frac{1}{2} \delta^{3}+2 \sqrt{2} \delta^{2}+6 \delta+4 \sqrt{2}\right) .
\end{aligned}
$$

Hence, we end the proof of the lemma.

Lemma 4 For every $u \in H^{1}(\mathbb{R})$, let $M=\max _{x \in \mathbb{R}}\{u(x)\}$. If

$$
\left|H_{1}[u]-H_{1}[\varphi]\right| \leq \delta(\delta+2 \sqrt{2})
$$

and

$$
\left|H_{2}[u]-H_{2}[\varphi]\right| \leq \delta\left(\frac{1}{2} \delta^{3}+2 \sqrt{2} \delta^{2}+6 \delta+4 \sqrt{2}\right) .
$$

for some $\delta$, then

$$
|M-1| \leq \sqrt{\delta\left(\delta^{3}+4 \sqrt{2} \delta^{2}+11 \delta+6 \sqrt{2}\right)} .
$$

Proof. In view of (31) in Lemma 2, the following inequality holds:

$$
H_{2}[u]-M^{2} H_{1}[u]+M^{4} \leq 0 .
$$

Define the polynomial $P$ by

$$
P(y)=H_{2}[u]-y^{2} H_{1}[u]+y^{4} .
$$

Using (25), $P(y)$ takes the form

$$
P_{0}(y)=H_{2}[\varphi]-y^{2} H_{1}[\varphi]+y^{4}=(y+1)^{2}(y-1)^{2},
$$

We calculate from (43) and (44) that

$$
P_{0}(M)=P(M)+M^{2}\left(H_{1}[u]-H_{1}[\varphi]\right)-\left(H_{2}[u]-H_{2}[\varphi]\right) .
$$

Note that, since $H_{1}[u]$ is near 2 and $H_{2}[u]$ is near 1,

$$
\frac{\sqrt{2}}{2}<\sqrt{\frac{H_{2}[u]}{H_{1}[u]}}<M,
$$


which along with (42), (44) and (45) yields

$$
(M-1)^{2} \leq M^{2}\left(H_{1}[u]-H_{1}[\varphi]\right)-\left(H_{2}[u]-H_{2}[\varphi]\right) .
$$

By (46) and the relation

$$
0 \leq M^{2} \leq \frac{H_{1}[u]}{2} \leq 1+\sqrt{2} \delta+\frac{1}{2} \delta^{2},
$$

we obtain

$$
\begin{aligned}
|M-1| & \leq \sqrt{M^{2}\left|H_{1}[u]-H_{1}[\varphi]\right|+\left|H_{2}[u]-H_{2}[\varphi]\right|} \\
& \leq \sqrt{\delta\left(\delta^{3}+4 \sqrt{2} \delta^{2}+11 \delta+6 \sqrt{2}\right)} .
\end{aligned}
$$

Hence, we end the proof of Lemma 4.

Proof of the Theorem 1 Since $H_{1}[u], H_{2}[u]$ are both conserved by the Equation (9), we have

$$
H_{1}[u(\cdot, t)]=H_{1}\left[u_{0}\right], H_{2}[u(\cdot, t)]=H_{2}\left[u_{0}\right], t \in(0, T) .
$$

We apply Lemma 3 to $u_{0}$ and to $\delta$. By the hypotheses of Lemma 4 are satisfied for $u(\cdot, t)$. Hence

$$
|u(\xi(t), t)-1| \leq \sqrt{\delta\left(\delta^{3}+4 \sqrt{2} \delta^{2}+11 \delta+6 \sqrt{2}\right)} .
$$

Combining (26) with Lemma 1, we find

$$
\begin{aligned}
& \| u(\cdot, t)-\left.\varphi(\cdot-\xi(t))\right|_{H^{1}(\mathbb{R})} ^{2} \\
& =H_{1}[u]-H_{1}[\varphi]+4(1-u(\xi, t)) \\
& \leq\left|H_{1}[u]-H_{1}[\varphi]\right|+4|1-u(\xi, t)| \\
& \leq \delta(\delta+2 \sqrt{2})+4 \sqrt{\delta\left(\delta^{3}+4 \sqrt{2} \delta^{2}+11 \delta+6 \sqrt{2}\right)}
\end{aligned}
$$

Based on the (49), for any $\epsilon>0$, we can take a $\delta(\epsilon)$ such that $\|u(\cdot, t)-\varphi(\cdot-\xi(t))\|_{H^{1}(\mathbb{R})}^{2}<\epsilon$. Therefore, we end the proof of 1 .

\section{Conclusion}

In this paper, we investigate the orbital stability of the peakons for a generalized Camassa-Holm equation $(\mathrm{gCH})$ with cubic nonlinearity. Using variable transformation, a planar dynamical system is obtained from the $\mathrm{gCH}$ equation. It is shown that the planar system has two heteroclinic cycles which correspond to two peakon solutions. We then prove that the peakons for the $\mathrm{gCH}$ equation are orbitally stable by using the method of Constantin and Strauss. The classical Camassa-Holm type equation models breaking waves, i.e. the solution, remain bounded while its slope becomes unbounded in finite time. In this paper, we do not investigate the breaking waves. We will study wave breaking for the $\mathrm{gCH}$ equation in the future.

\section{Acknowledgements}

This work is supported by the National Natural Science Foundation of China 
(No. 11671107), Guangxi Natural Science Foundation of China (No. 2015GXN SFGA139004) and Program for Innovation Project of GUET Graduate Education

(No. 2019YCXS080).

\section{Conflicts of Interest}

The authors declare no conflicts of interest regarding the publication of this paper.

\section{References}

[1] Camassa, R. and Holm, D.D. (1993) An Integrable Shallow Water Equation with Peaked Solitons. Physical Review Letters, 71, 1661-1664. https://doi.org/10.1103/PhysRevLett.71.1661

[2] Camassa, R., Holm, D.D. and Hyman, J. (1994) A New Integrable Shallow Water Equation. Advances in Applied Mechanics, 31, 1-33. https://doi.org/10.1016/S0065-2156(08)70254-0

[3] Constantin, A. and Escher, J. (1998) Wave Breaking for Nonlinear Nonlocal Shallow Water Equations. Acta Mathematica, 181, 229-243. https://doi.org/10.1007/BF02392586

[4] Constantin, A. (2000) Global Existence of Solutions and Wave Breaking Waves for a Shallow Water Equation: A Geometric Approach. Annales de Pinstitut Fourier (Grenoble), 50, 321-362. https://doi.org/10.5802/aif.1757

[5] Constantin, A. and Escher, J. (1998) Global Existence and Blow-Up for a Shallow Water Equation. Annali della Scuola Normale Superiore di Pisa, 26, 303-328.

[6] Constantin, A. and Escher, J. (2000) On the Blow-Up Rate and the Blow-Up Set of Breaking Waves for a Shallow Water Equation. Mathematische Zeitschrift, 33, 75-91. https://doi.org/10.1007/PL00004793

[7] Alber, M.S., Comassa, R., Holm, D.D. and Marsden, J.E. (1994) The Geometry of Peaked Solitons and Billiard Solutions of a Class of Integrable PDE's. Letters in Mathematical Physics, 32, 137-151. https://doi.org/10.1007/BF00739423

[8] Cao, C.S., Holm, D.D. and Titi, E.S. (2004) Traveling Wave Solutions for a Class of One-Dimensional Nonlinear Shallow Water Wave Models. Journal of Dynamics and Differential Equations, 16, 167-178. https://doi.org/10.1023/B:JODY.0000041284.26400.d0

[9] Fisher, M. and Schff, J. (1999) The Camassa-Holm Equation: Conserved Quantities and the Initial Value Problem. Physics Letters A, 259, 271-376.

https://doi.org/10.1016/S0375-9601(99)00466-1

[10] Fuchssteiner, B. and Fokas, A.S. (1982) Symplectic Structures, Their Backlund Transformations and Hereditary Symmetries. Journal of Physics D, 4, 47-66. https://doi.org/10.1016/0167-2789(81)90004-X

[11] Anco, S.C. and Recio, E. (2019) A General Family of Multi-Peakon Equations and Their Properties. Journal of Physics A: Mathematical and Theoretical, 52, 125-203. https://doi.org/10.1088/1751-8121/ab03dd

[12] Constantin, A. and Strauss, W. (2000) Stability of Peakons. Communications on Pure and Applied Mathematics, 53, 603-610. https://doi.org/10.1002/(SICI)1097-0312(200005)53:5<603::AID-CPA3>3.3.CO;2-C

[13] Constantin, A. and Molinet, L. (2001) Orbital Stability of Solitary Waves for a Shallow Water Equation. Journal of Physics D, 157, 75-89. 
https://doi.org/10.1016/S0167-2789(01)00298-6

[14] Yin, J.L. and Tian, L.X. (2010) Stability of Peakons and Linear Dispersion Limit for the Periodic Dullin-Gottwald-Holm Equation. Journal of Mathematical Physics, 51, Article ID: 194501. https://doi.org/10.1063/1.3120914

[15] Lin, Z.W. and Liu, Y. (2009) Stability of Peakons for the Degasperis-Procesi Equation. Communications on Pure and Applied Mathematics, 62, 125-146. https://doi.org/10.1002/cpa.20239

[16] Liu, X.C., Liu, Y. and Qu, C. (2014) Orbital Stability of the Train of Peakons for an Integrable Modified Camassa-Holm Equation. Advances in Mathematics, 255, 1-37. https://doi.org/10.1016/j.aim.2013.12.032

[17] Qu, C., Liu, X.C. and Liu, Y. (2013) Stability of Peakons for an Integrable Modified Camassa-Holm Equation with Cubic Non-Linearity. Communications in Mathematical Physics, 322, 967-997. https://doi.org/10.1007/s00220-013-1749-3

[18] Guo, Z., Liu, X., Liu, X. and Qu, C. (2019) Stability of Peakons for the Generalized Modified Camassa-Holm Equation. Journal of Differential Equations, 266, 7749-7779. https://doi.org/10.1016/j.jde.2018.12.014

[19] Liu, X.C., Yue, L. and Qu, C. (2014) Stability of Peakons for the Novikov Equation. Journal de Mathématiques Pures et Appliquées, 101, 172-187. https://doi.org/10.1016/j.matpur.2013.05.007

[20] Chen, R.M., Liu, X.C. and Liu, Y. (2016) Stability of the Camassa-Holm Peakons in the Dynamics of a Shallow-Water-Type System. Calculus of Variations, 55, 34-55. https://doi.org/10.1007/s00526-016-0972-0

[21] Gui, G., Liu, Y., Olver, P.J., et al. (2013) Wave-Breaking and Peakons for a Modified Camassa-Holm Equation. Communications in Mathematical Physics, 319, 731-759. https://doi.org/10.1007/s00220-012-1566-0

[22] Ouyang, Z.Y., Zheng, S. and Liu, Z.R. (2008) Orbital Stability of Peakons with Nonvanishing Bundary for $\mathrm{CH}$ and $\mathrm{CH}-\gamma$ Equation. Physics Letters A, 47, 7046-7050.

[23] Liu, X.X. and Yin, Z.Y. (2011) Local Well-Posedness and Stability of Peakons for a Generalized Dullin-Gottwald-Holm Equation. Nonlinear Analysis, 74, 2497-2507.

https://doi.org/10.1016/j.na.2010.12.005

[24] Liu, X.X. (2018) Orbital Stability of Peakons for a Modified Camassa-Holm Equation with Higher-Order Nonlinearity. Discrete \& Continuous Dynamical Systems, 38, 5505-5521. https://doi.org/10.3934/dcds.2018242

[25] Liu, X.C., Yue, L., Olver, P.J. and Qu, C. (2014) Orbital Stability of Peakons for a Generalization of the Modified Camassa-Holm Equation. Nonlinearity, 27, 2297-2319. https://doi.org/10.1088/0951-7715/27/9/2297

[26] Lenells, J. (2004) Stability of Periodic Peakons. International Mathematics Research Notices, 10, 485-499. https://doi.org/10.1155/S1073792804132431

[27] Chen, R.M., Lenells, J. and Liu, Y. (2010) Stability of the $\mu$-Camassa-Holm Peakons. Journal of Nonlinear Science, 23, 97-112. https://doi.org/10.1007/s00332-012-9141-6

[28] Yin, J.L. and Fan, X.H. (2010) Orbital Stability of Floating Periodic Peakons for the Camassa-Holm Equation. Nonlinear Analysis, 11, 4021-4026. https://doi.org/10.1016/j.nonrwa.2010.03.008

[29] Liu, Y., Qu, C. and Zhang, Y. (2013) Stability of Periodic Peakons for the Modified $\mu$-Camassa-Holm Equation. Journal of Physics D, 250, 66-74. 
https://doi.org/10.1016/j.physd.2013.02.001

[30] Qu, C., Zhang, Y., Liu, X.C. and Liu, Y. (2014) Orbital Stability of Periodic Peakons to a Generalized $\mu$-Camassa-Holm Equation. Archive for Rational Mechanics and Analysis, 211, 593-617. https://doi.org/10.1007/s00205-013-0672-2 